\title{
On the Relation between Finitely and Infinitely Repeated Games with Incomplete Information ${ }^{1}$ )
}

\author{
By SHMUEL $Z_{\mathrm{AmIR}}{ }^{2}$ )
}

\begin{abstract}
For a class of repeated two-person zero-sum games with incomplete intormation it was proved by AUMANN and MASCHLER that $\lim v_{n}$ exists, $v_{n}$ being the value of the game with $n$ repetitions. As for the speed of convergence AUMANN and MASCHLER showed that the error term $\delta_{n}=\left|v_{n}-\lim v_{n}\right|$ is bounded from above by $c / \sqrt{n}$ for some positive constant $c$. Both results have been generalized by MERTENS and ZAMIR. It is shown in this paper that the above mentioned theorem about the speed of convergence is sharp in the sense that there are games in which $\delta_{n} \geq c^{\prime} \sqrt{n}$ for some positive constant $c^{\prime}$. However there are games for which $\delta_{n}$ is of a lower order of magnitude, for instance $c^{\prime}(\log n) / n \leq \delta_{n} \leq$ $c(\log n) / n$ or $c^{\prime} / n \leq \delta_{n} \leq c / n$. Sufficient conditions are given here for games to belong to one of these categories as well as examples of games from each category.
\end{abstract}

\section{Introduction}

Following the introduction by HARSANYI of the concept of games with incomplete information, AUMANN and MASCHLER and later on MERTENS and ZAMIR treated a certain situation in which a two-person zero-sum game is repeated a large number of times by the same players. The main feature of such a situation is the possibility of each player to collect information, unknown to him but known to the other player, by watching the moves of his opponent. Consequently, each player must be aware of the fact that by his moves he is revealing information to his opponent that may be used against him in a further stage. Optimal strategies of the players in such a multistage game must therefore reflect an optimal speed of revealing information. In the mathematical model this process of revealing and collecting information shows up implicitly through $v_{n}$ the value of the game with $n$ repetitions. (For mathematical convenience $v_{n}$ is defined to be "the value per stage" i.e. the value of the supergame composed of $n$ stages, divided by $n$ ). The main result by AUMANN and MASCHLER (as well as in its generalization by MERTENS and ZAMIR) is that $v_{n}$ converges (as $n \rightarrow \infty$ ) to some constant $v$ which may be called the value of the infinite game. The error term $\delta_{n}=\left|v_{n}-v\right|(n=1,2, \ldots)$ gives the speed of convergence of $v_{n}$ to $v$ which is determined implicitly by the flow of information throughout the stages of the game, as the two players play

\footnotetext{
1) Part of this paper is based on a chapter of the author's Ph. D. thesis done at the Institute of Mathematics at the Hebrew University of Jerusalem.

$\left.{ }^{2}\right)$ SHMUEL Zamir, Department of Mathematics, University of California, Los Angeles, 405 Hilgard Avenue, Los Angeles, California 90024.
} 
optimally. Since usually $v$ can be computed with a reasonable effort while $v_{n}$ is practically impossible to compute for a very large $n, \delta_{n}$ has also a very practical importance: To give us the error involved in approximating $v_{n}$ by $v$.

It is the purpose of this paper to investigate the error term $\delta_{n}$ more closely. By AumAnN and MASCHLER and by MerTEns and ZAMIR it was shown that (for the games treated there) $\delta_{n} \leq c / \sqrt{n}$ for some constant $c$. Our first task will be to show that this is a sharp estimation for the order of magnitude of $\delta_{n}$, by demonstrating an example of a game in which $\delta_{n} \geq c^{\prime} / \sqrt{n}$ for some positive constant $c^{\prime}$. In such a game $v_{n}$ converges to $v$ at the slowest (order of magnitude) speed possible. There are however games in which the convergence is much faster e.g. $c^{\prime}(\log n) / n \leq \delta_{n} \leq c(\log n) / n$ or even $c^{\prime} / n \leq \delta_{n} \leq c / n$. We shall give two sufficient conditions, one for each of the above properties, and we shall also give examples of such games.

Although the works of HARSANY, Aumann and MASCHLER, and Mertens and ZAMIR are very relevant and very important background for this research, we tried to make this paper self contained. Familiarity with the above mentioned references is not a requirement for understanding this paper.

I am indebted to R. J. AuMANN for many helpful discussions and to M. MASCHLER for his advice about the representation of the material. I would also like to thank A. PAZY and T. S. FERGUSON for valuable remarks on the proof of Lemma 3.

\section{The Class of Games}

For the purposes of this paper it will be sufficient for us to look at a subfamily of the games considered by MERTENS and ZAMIR, namely the subfamily of games with lack of information on one side. These are the games treated by AUMANN and MASCHLER and can be described as follows:

Let $A^{1}, \ldots, A^{k},(k \geq 2)$, be $l \times M$ matrices viewed as two-person zero-sum games. For each $p$ in the simplex $P=\left\{p=\left(p^{1}, \ldots, p^{k}\right) \mid p^{\nu} \geq 0, v=1, \ldots, k\right.$, $\left.\sum_{1}^{k} p^{\nu}=1\right\}$ and for each positive integer $n$, consider the game $\Gamma_{n}(p)$ played as follows: Chance chooses one of the $k$ games $\left\{A^{v}\right\}$ assigning to $A^{v}$ the probability $p^{v}$. Player I is then informed of chance's choice but player II is not. The game chosen by chance is then played $n$ times; after each play both players are informed of the move made by the other player but not of the payoff. (Player I can, of course, deduce the payoff from the strategy choices made by himself and the other player, since he knows which game is actually being played.) The payoff in $\Gamma_{n}(p)$ is defined to be the sum of the payoffs in the $n$ individual plays of the game chosen by chance, divided by $n$. Both players of course know the rules of $\Gamma_{n}(p)$ as described above. 
We shall describe the specifications of such a game schematically by:

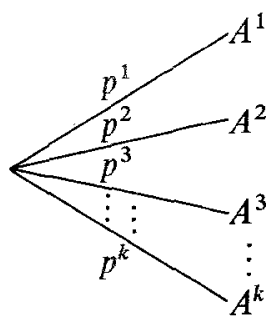

Let $v_{n}(p)$ denote the (minimax) value of $\Gamma_{n}(p) .\left(v_{n}(p)\right.$ surely exists for all $p \in P$ since $\Gamma_{n}(p)$ itself is a zero-sum two-person game. Furthermore, since the payoffs in $\Gamma_{n}(p)$ are linear in $p, v_{n}(p)$ is continuous on $P$.) Let $\Delta(p)$ denote the two-person zero-sum game whose matrix is $\sum_{1}^{k} p^{v} A^{v}$, and let $u(p)$ be its value. The game $\Delta(p)$ is actually the original game with the modification that player I also is not informed which $A^{v}$ was chosen by chance. Or equivalently $\Delta(p)$ is the original game in which player $I$ behaves as if he did not know the choice of chance and therefore the strategy he uses is independent of his 'type', $v$. As far as the question or revealing information is concerned a strategy of player $\mathrm{I}$ in $\Delta(p)$ represents an extreme behavior in which he disregards the additional information he has (and therefore conceals it from player II). Let $u^{*}(p)$ be the concavification of $u(p)$, i.e. $u^{*}$ is the smallest concave function on $P$ such that $u^{*}(p) \geq u(p)$ for all $p \in P$.

Theorem 1. (AUMANN-MASCHLER)

(i) For all $p \in P, \lim _{n \rightarrow \infty} v_{n}(p)$ exists and is equal to $u^{*}(p)$.

(ii) $\delta_{n}=\left|v_{n}(p)-u^{*}(p)\right| \leq c(p) / \sqrt{n}$, where $c(p)$ is a constant dependent on $p$.

(This follows, of course, from the more general results of MERTENS and ZAMIR;

(i) is a special case of Theorem 2.1 while (ii) is a special case of Theorem 4.5).

It will be very convenient to use throughout this paper the following terminology and notation which is slightly different from the usual one.

\section{Definition}

Let $\left\{a_{n}\right\}$ and $\left\{b_{n}\right\}$ be two sequences of nonnegative real numbers. They will be said to be of the same order of magnitude if there are $c_{1}>0, c_{2}>0$ such that $c_{2} b_{n} \leq a_{n} \leq c_{1} b_{n}$ for $n=1,2, \ldots$. We shall denote this by $a_{n}=0^{*}\left(b_{n}\right)$, (or $b_{n}=0^{*}\left(a_{n}\right)$ ). (Compare to $a_{n}=0\left(b_{n}\right)$ which means: $a_{n} / b_{n}$ is bounded.)

The relation between $\delta_{n}(p)$ and $1 / \sqrt{n}$ has the following probabilistic interpretation: Let us specialize to the case $k=2, l=2$ and $u^{*}(p)=u(p)$; a similar discussion applies in general. Since $\lim v_{n}(p)=u^{*}(p)=u(p)$, in the limit as $n \rightarrow \infty$ player I can not obtain more than $u(p)$ which is what he can guarantee by disregarding his additional information and playing identically in both games. Nevertheless player I can generally obtain more than $u(p)$ in $\Gamma_{n}(p)$ for any finite $n$. In order to do this he has to play differently in the two possible games. In other 
words he has to deviate from an extreme non-revealing strategy. How much can he deviate and how much can he gain by this deviation? Let $(s, 1-s)$ be player I's optimal mixed strategy in $\Delta(p)$ (i.e. I plays his first pure strategy with probability $s$ and his second pure strategy with probability $1-s(0 \leq s \leq 1))$. By its definition, when this strategy is played repeatedly in the $n$ stages of $\Gamma_{n}(p)$ player I guarantees $u(p)$. If player I in fact does this, the actual choice of pure strategy made by him can be regarded as a Bernoulli trial with probabilities $s, 1-s$. The proportion of times that the first pure strategy is played in $n$ such trials distributes approximately normally around the mean $s$ with a standard deviation of the order of $1 / \sqrt{n}$. Therefore if player I wishes to "cheat" player II by making use of the additional information (i.e. playing different mixed strategies in the two possible games) without enabling him to detect it, he may do this in such a way that this proportion will fall within the limits of few standard deviations i.e. $c / \sqrt{n}$ from s. Any deviation of higher order will be detected and used by player II to hold the payoff to a number smaller than $u(p)$. Clearly a deviation of order not higher than $1 / \sqrt{n}$ from the optimal strategy $(s, 1-s)$ will make a deviation in the payoff in $\Gamma_{n}(p)$ which is also of order not higher than $1 / \sqrt{n}$.

The existence of games with $\delta_{n}=0^{*}(1 / \sqrt{n})$, which is the main result of this paper, implies that there are games in which player I can exhaust the whole probabilistic deviation mentioned above. In other games he may be able to exhaust only a small part of it such as $0^{*}\left(\frac{\log n}{n}\right)$ or $0^{*}\left(\frac{1}{n}\right)$.

\section{A Recursion Formula}

We start with a useful formula giving $v_{n+1}(p)$ in terms of $v_{n}(p)$. Let $\Sigma=\{\sigma=$ $\left.\left(s_{1}, \ldots, s_{l}\right) \mid s_{i} \geq 0, i=1, \ldots, l, \Sigma_{1}^{l} s_{i}=1\right\}$ and $T=\left\{\tau=\left(t_{1}, \ldots, t_{m}\right) \mid t_{j} \geq 0, j=\right.$ $\left.1, \ldots, m ; \Sigma_{1}^{m} t_{j}=1\right\}$ be the sets of mixed strategies of player I and II respectively in $\left\{A^{v}\right\}$. Denote by $\Sigma^{*}$ the $k$-th cartesian power of $\Sigma$. i.e. $\Sigma^{*}=\Sigma^{k}=\Sigma \times \cdots \times \Sigma$. Finally let $\mathrm{e}_{i}$ for $i=1, \ldots, m$, be the $i$-th unit column $\left(\begin{array}{c}0 \\ \vdots \\ 0 \\ 1 \\ 0 \\ \vdots \\ 0\end{array}\right)$ in $T$.

\section{Lemma 1.}

For every $p \in P$ and for $n=1,2, \ldots$.

$$
v_{n+1}(p)=\frac{1}{n+1} \max _{\sigma^{*} \in \Sigma^{*}}\left\{\min _{1 \leq j \leq m} \sum_{\nu=1}^{k} p^{v} \sigma^{v} A^{v} \mathrm{e}_{j}+n \sum_{\substack{i=1 \\ \bar{s}_{i} \neq 0}}^{l} \bar{s}_{i} v_{n}\left(p_{i}\right)\right\}
$$


where: $\sigma^{*}=\left(\sigma^{1}, \ldots, \sigma^{k}\right), \sigma^{v} \in \Sigma, v=1, \ldots, k$

$$
\left\{\begin{array}{l}
\bar{\sigma}=\left(\bar{s}_{1}, \ldots, \bar{s}_{l}\right)=\sum_{v=1}^{k} p^{v} \sigma^{v} \text { and for each } i \text { such that } \bar{s}_{i} \neq 0: \\
p_{i}=\left(p_{i}^{1}, \ldots, p_{i}^{k}\right) ; p_{i}^{v}=\frac{p^{v} s_{i}^{v}}{\bar{s}_{i}} \quad v=1, \ldots, k, i=1, \ldots, l .
\end{array}\right.
$$

Proof.

The part concerning the first stage in any strategy of player $\mathrm{I}$ in $\Gamma_{n+1}(p)$ is an element $\sigma^{*}=\left(\sigma^{1}, \ldots, \sigma^{k}\right)$ of $\Sigma^{*}$ and it says to play $\sigma^{v}$ if chance chooses $A^{v}$, $v=1, \ldots, k$. With a strategy starting this way player I's expected payoff for the first stage will be at least $\min _{1 \leq j \leq m} \sum_{v=1}^{k} p^{v} \sigma^{v} A^{v} \mathrm{e}_{j}$. Making use of the minimax theorem we can assume that player II knows the strategy chosen by player I in $\Gamma_{n+1}(p)$. In particular we assume that player II knows $\sigma^{*}$. After the first stage as he is informed which pure strategy $i$ was chosen by player I, player II calculates the probability vector $p_{i}=\left(p_{i}^{1}, \ldots, p_{i}^{k}\right)$ where $p_{i}^{v}$ is his new conditional probability for the event "chance has chosen $A^{v "}$, given $i, \sigma^{*}$ and $p$. Clearly $p_{i}$ is given by (2) and the probability of obtaining $p_{i}$ is the total probability that player I chooses $i$ which is $\bar{s}_{i}$.

After the first stage, therefore, the situation is equivalent to the following: Chance has chosen one of the games $\left\{A^{v}\right\}$ according to the probability distribution $p_{i}$. Player I is informed which game was chosen and player $I$ is not. The game chosen by chance has to be played $n$ times. In other words, after the first stage, the players will face one of the games $\Gamma_{n}\left(p_{1}\right) \ldots \Gamma_{n}\left(p_{l}\right)$ (with probabilities $\bar{s}_{1}, \ldots, \bar{s}_{l}$ respectively, and hence $p_{i}$ has to be defined only for $i$ s.t. $\bar{s}_{i} \neq 0$ ). By playing optimally in $\Gamma_{n}\left(p_{i}\right)$ $(i=1, \ldots, l)$, player $\mathrm{I}$ guarantees an expected payoff of $n v_{n}\left(p_{i}\right)$ for the coming $n$ stages. Thus, by maximizing with respect to $\sigma^{*}$ we have:

$$
v_{n+1}(p) \geq \frac{1}{n+1} \max _{\sigma^{*} \in \Sigma^{*}}\left\{\min _{\substack{1 \leq j \leq m \\ 1 \leq 1}} \sum_{v=1}^{k} p^{v} \sigma^{v} A^{v} \mathrm{e}_{j}+n \sum_{\substack{i=1 \\ s_{i} \neq 0}}^{l} \bar{s}_{i} v_{n}\left(p_{i}\right)\right\}
$$

On the other hand, knowing $\sigma^{*}$, player II can guarantee

$$
\frac{1}{n+1}\left\{\min _{1 \leq j \leq m} \sum_{v=1}^{k} p^{v} \sigma^{v} A^{v} \mathrm{e}_{j}+n \sum_{\substack{i=1 \\ \bar{s}_{i} \neq 0}}^{t} \bar{s}_{i} v_{n}\left(p_{i}\right)\right\}
$$

and since by the minimax theorem we can assume that he knows $\sigma^{*}$, we get also the opposite inequality of (3) and hence the lemma is proved.

\section{Notation.}

Since throughout this paper we will use summations of the form $\sum_{\substack{i=1 \\ \bar{s}_{i} \neq 0}}^{l} \bar{s}_{i} \varphi_{i}$ where $\varphi_{i}$ is usually not defined when $\bar{s}_{i}=0$ we will write this in short as $\sum_{i=1}^{l} \bar{s}_{i} \varphi_{i}$ and
keep in mind that the summation is only over $i$ such that $\bar{s}_{i} \neq 0$. 


\section{A Game with $\delta_{n}=0^{*}(1 / \sqrt{n})$}

Consider the game: $\sum_{p^{\prime}}^{p} A^{1}$ where:

$$
A^{1}=\frac{L}{R}\left(\begin{array}{rr}
3 & -1 \\
-3 & 1
\end{array}\right) \quad A^{2}=\frac{L}{R}\left(\begin{array}{rr}
2 & -2 \\
-2 & 2
\end{array}\right)
$$

(We will often use in this paper the notation $p^{\prime}$ for $1-p$ ). $\Delta(p)$ is the game: $\left(\begin{array}{rr}3 p+2 p^{\prime} & -p-2 p^{\prime} \\ -3 p-2 p^{\prime} & p+2 p^{\prime}\end{array}\right)$ and its value is $u(p)=0$ for $0 \leq p \leq 1$. So by Theorem 1 :

$$
v(p)=u^{*}(p)=u(p)=0 \text { for } 0 \leq p \leq 1 .
$$

$\Gamma_{1}(p)$ is the game:

$$
\begin{aligned}
& L L \\
& L R \\
& R L \\
& R R
\end{aligned}\left(\begin{array}{rr}
3 p+2 p^{\prime} & -p-2 p^{\prime} \\
3 p-2 p^{\prime} & -p+2 p^{\prime} \\
-3 p+2 p^{\prime} & p-2 p^{\prime} \\
-3 p-2 p^{\prime} & p+2 p^{\prime}
\end{array}\right)
$$

( $L L$ is the strategy of player I: $L$ in $A^{1}$ and $L$ in $A^{2} ; L R, R L$ and $R R$ are interpreted similarly). The value of this game is easily computed to be:

$$
v_{1}(p)=\left\{\begin{array}{ll}
p & 0 \leq p \leq \frac{1}{2} \\
p^{\prime} & \frac{1}{2} \leq p \leq 1
\end{array}=\min \left(p, p^{\prime}\right)\right.
$$

Theorem 2.

In the above described game:

$$
v_{n}(p) \geq p p^{\prime} \sqrt{n} ; \quad 0 \leq p \leq 1 \text { for } n=1,2, \ldots .
$$

Proof.

In the special case under consideration $\Sigma=\left\{\left(s, s^{\prime}\right) \mid 0 \leq s \leq 1\right\}, \Sigma^{*}=\Sigma \times \Sigma=$ $\left\{\left(s, s^{\prime}\right),\left(t, t^{\prime}\right) \mid 0 \leq s \leq 1 ; 0 \leq t \leq 1\right\}$. It is more convenient to use $L$ and $R$ for the pure strategies of player I and hence we shall write $p_{L}$ and $p_{R}$ instead of $p_{1}$ and $p_{2}$. So we can rewrite (2) for our game as:

$$
\left\{\begin{array}{l}
\left(\bar{s}, \bar{s}^{\prime}\right)=\left(p s+p^{\prime} t, p s^{\prime}+p^{\prime} t^{\prime}\right) \\
p_{L}=\frac{p s}{\bar{s}} ; \quad p_{R}=\frac{p s^{\prime}}{\bar{s}^{\prime}} .
\end{array}\right.
$$

Let us now prove (4) by induction on $n$ : For $n=1, v_{1}(p)=\min \left(p, p^{\prime}\right) \geq p p^{\prime}$ in accordance with (4). Assume that (4) is true for $n$ and let us prove it for $n+1$. For $p=0$ or $p^{\prime}=0$ clearly $v_{n+1}(p)=0$ in accordance with (4) so assume $p p^{\prime} \neq 0$.

By the recursion formula (1) and by (5) we have

$$
\begin{aligned}
v_{n+1}(p)= & \frac{1}{n+1} \max _{\substack{0 \leq s \leq 1 \\
0 \leq t \leq 1}}\left\{\operatorname { m i n } \left[p\left(3 s-3 s^{\prime}\right)+p^{\prime}\left(2 t-2 t^{\prime}\right) ; p\left(-s+s^{\prime}\right)\right.\right. \\
& \left.\left.+p^{\prime}\left(-2 t+2 t^{\prime}\right)\right]+n\left(\bar{s} v_{n}\left(p_{L}\right)+\bar{s}^{\prime} v_{n}\left(p_{R}\right)\right)\right\}
\end{aligned}
$$


Let us restrict $s$ and $t$ by the relation

Then:

$$
p\left(s-s^{\prime}\right)=p^{\prime}\left(t^{\prime}-t\right) \quad \text { which is } \quad t=\frac{1}{2}-\frac{p\left(s-s^{\prime}\right)}{2 p^{\prime}} .
$$

$$
\min \left[p\left(3 s-3 s^{\prime}\right)+p^{\prime}\left(2 t-2 t^{\prime}\right) ; p\left(-s+s^{\prime}\right)+p^{\prime}\left(-2 t+2 t^{\prime}\right)\right]=p\left(s-s^{\prime}\right) \text {. }
$$

By (5) and (7) we have

$$
\bar{s}=\bar{s}^{\prime}=\frac{1}{2} ; p_{L}=2 p s ; p_{R}=2 p s^{\prime} .
$$

From (6) we get by (8) and (9)

$$
v_{n+1}(p) \geq \max _{\substack{0 \leq s \leq 1 \\ 0 \leq \frac{1}{2}-\frac{p\left(s-s^{\prime}\right)}{2 p^{\prime}} \leq 1}} \frac{1}{n+1}\left\{p\left(s-s^{\prime}\right)+\frac{n}{2}\left(v_{n}(2 p s)+v_{n}\left(2 p s^{\prime}\right)\right)\right\}
$$

and using the induction hypothesis we have:

$$
v_{n+1}(p) \geq \max _{\substack{0 \leq s \leq 1 \\ 0 \leq \frac{1}{2}-\frac{p\left(s-s^{\prime}\right)}{2 p^{\prime}} \leq 1}} \frac{1}{n+1}\left\{p\left(s-s^{\prime}\right)+\frac{\sqrt{n}}{2}\left((2 p s)(2 p s)^{\prime}+\left(2 p s^{\prime}\right)\left(2 p s^{\prime}\right)^{\prime}\right)\right\}
$$

As a result of simple maximization in (10) we get the following values for $s$ :

Case a.

For $(1 / 4 \sqrt{n}) \leq p \leq 1-(1 / 4 \sqrt{n})$ we take $s_{0}=\frac{1}{2}+(1 / 8 p \sqrt{n})$ (which will be in the domain of maximization) and we easily get by (10):

$$
\begin{aligned}
v_{n+1}(p) & \geq \frac{1}{n+1}\left\{(1 / 4 \sqrt{n})+\frac{\sqrt{n}}{2}\left(2 p p^{\prime}-\frac{1}{8 n}\right)\right\} \\
& =\frac{1}{n+1}\left\{p p^{\prime} \sqrt{n}+(1 / 4 \sqrt{n})-(1 / 16 \sqrt{n})\right\} \\
& \geq \frac{1}{n+1}\left\{p p^{\prime} \sqrt{n}+(1 / 8 \sqrt{n})\right.
\end{aligned}
$$

and since $4 p p^{\prime} \leq 1$ we get finally

$$
v_{n+1}(p) \geq \frac{p p^{\prime}}{n+1}(\sqrt{n}+(1 / 2 \sqrt{n})) \geq p p^{\prime} / \sqrt{n+1} .
$$

Case b.

$0 \leq p<(1 / 4 \sqrt{n})$. In this case we take $s_{0}=1$ and then by $(10)$ :

$$
\begin{aligned}
v_{n+1}(p) & \geq \frac{1}{n+1}\left\{p+\frac{\sqrt{n}}{2} \cdot 2 p(1-2 p)\right\} \\
& =\frac{1}{n+1}\left\{p p^{\prime} \sqrt{n}+p(1-p \sqrt{n})\right\} \\
& \geq \frac{1}{n+1}\left\{p p^{\prime} \sqrt{n}+p \cdot \frac{3}{4}\right\} \geq p p^{\prime} \sqrt{n+1}
\end{aligned}
$$


Case c.

$1-(1 / 4 \sqrt{n})<p \leq 1$. The result in this case follows either from case $b$ by symmetry arguments (the functions $u(p)$ and $v_{n}(p)$ satisfy $u(p)=u\left(p^{\prime}\right), v_{n}(p)=v_{n}\left(p^{\prime}\right)$ ) or directly by choosing $s_{0}=\frac{1}{2}-\frac{p^{\prime}}{2 p}$. Hence the theorem is proved.

Corollary.

By combining Theorems 1 and 2 we have that the error term $\delta_{n}(p)$ of the game under consideration satisfies $p p^{\prime} / \sqrt{n} \leq \delta_{n}(p) \leq c / \sqrt{n}$ for some constant $c$, and hence $\delta_{n}=0^{*}(1 / \sqrt{n})$. In other words $0(1 / \sqrt{n})$ is in the general case the least upper bound for the order of magnitude of $\delta_{n}$.

\section{A Sufficient Condition for $\delta_{n}=0\left(\frac{\log n}{n}\right)$}

After the example in the last section establishing the existence of games with error term equal to $0^{*}(1 / \sqrt{n})$, we turn in this section to games with a much smaller error term namely $\delta_{n}=0\left(\frac{\log n}{n}\right)$.

For any $\Gamma_{n}(p) ; n=1,2, \ldots$ we will denote $\lim v_{n}(p)$ by $v(p)$. (Hence by Theorem 1 $v(p)=u^{*}(p)$.) For the statement and the proof of the next theorem it is convenient to consider $v(p)$ as a function of $k-1$ variables, say $p^{1}, \ldots, p^{k-1}$ (putting $\left.p^{k}=1-\sum_{1}^{k-1} p^{i}\right)$. For any $p \in P$ we denote by $v^{\prime}(p)$ the gradient $\left(\begin{array}{c}\frac{\partial v}{\partial p^{1}} \\ \vdots \\ \frac{\partial v}{\partial p^{k-1}}\end{array}\right)$ of $v$ at the point $p$ and by $v^{\prime \prime}(p)$ the $(k-1) \times(k-1)$ matrix of second derivatives $\left(\frac{\partial^{2} v}{\partial p^{i} \partial p^{j}}\right)$, $1 \leq i \leq k-1,1 \leq j \leq k-1$, evaluated at $p$. For any vector $q$ we denote by $\tilde{q}$ its transposition and by $\|q\|$ its Euclidean norm in the corresponding Euclidean space. (Usually this will be $E^{k}$ and occasionally $E^{k-1}$. No confusion may arise since it will be always clear to which space $q$ belongs and hence what norm is meant by $\|q\|$.)

\section{Theorem 3.}

If $\sum_{p^{k}}^{p^{1}} A^{k}$ is a game with incomplete information with value $v(p)$ such that $v^{\prime}(p)$ and $v^{\prime \prime}(p)$ exist for all $p \in P$ and there exist a constant $\eta>0$ such that $u v^{\prime \prime}(p) \tilde{u} \leq-\eta$ for all $p \in P$ and all $u \in E^{k-1},\|u\|=1$, then $\delta_{n}=0\left(\frac{\log n}{n}\right)$. 
For the proof of this theorem we need the following two lemmas:

\section{Lemma 2.}

Let ${\stackrel{p^{k}}{p^{k}} A^{1}}_{A^{k}}^{A^{1}}$ be a game with incomplete information. For any $\sigma^{*}=$ $\left(\sigma^{1}, \ldots, \sigma^{k}\right) \in \Sigma^{*}$

$$
\min _{1 \leq j \leq m} \sum_{v=1}^{k} p^{v} \sigma^{v} A^{v} \mathrm{e}_{j} \leq u(p)+c \sum_{i=1}^{l} \bar{s}_{i}\left\|p-p_{i}\right\|
$$

where $\bar{s}_{i}$ and $p_{i}$ are given by (2), and $c$ is a constant.

Proof.

Denote $A^{v}=\left(a_{i j}^{v}\right), a=\max \left\{a_{i j}^{v} \mid 1 \leq i \leq l, 1 \leq j \leq m, 1 \leq v \leq k\right\}$ and $c=a k ;$ then:

$$
\begin{aligned}
\min _{1 \leq j \leq m} \sum_{v=1}^{k} p^{v} \sigma^{v} A^{v} \mathrm{e}_{j} & =\min _{1 \leq j \leq m}\left\{\sum_{v=1}^{k} p^{v} \bar{\sigma} A^{v} \mathrm{e}_{j}+\sum_{v=1}^{k} p^{v}\left(\sigma^{v}-\bar{\sigma}\right) A^{v} \mathrm{e}_{j}\right\} \\
& \leq \min _{1 \leq j \leq m} \bar{\sigma}\left(\sum_{v=1}^{k} p^{v} A^{v}\right) \mathrm{e}_{j}+a \sum_{v=1}^{k} p^{v} \sum_{i=1}^{l}\left|s_{i}^{v}-\bar{s}_{i}\right|
\end{aligned}
$$

Now recall that $\sum_{v=1}^{k} p^{v} A^{v}$ is the payoff matrix of the game $\Delta(p), \bar{\sigma}$ is a strategy of player $\mathrm{I}$ in this game. Therefore the first term is not more than the value $u(p)$. Hence

$$
\min _{1 \leq j \leq m} \sum_{v=1}^{k} p^{v} \sigma^{v} A^{v} \mathrm{e}_{j} \leq u(p)+a \sum_{v=1}^{k} p^{\nu} \sum_{i=1}^{l}\left|s_{i}^{v}-\bar{s}_{i}\right|
$$

Using (2) we obtain:

$$
\begin{aligned}
\min _{1 \leq j \leq m} \sum_{v=1}^{k} p^{v} \sigma^{v} A^{v} \mathrm{e}_{j} & \leq u(p)+a \sum_{i=1}^{l} \sum_{v=1}^{k} \bar{s}_{i}\left|\frac{p^{v} s_{i}^{v}}{\bar{s}_{i}}-p^{v}\right| \\
& =u(p)+a \sum_{i=1}^{l} \bar{s}_{i} \sum_{v=1}^{k}\left|p_{i}^{v}-p^{v}\right| \\
& \leq u(p)+a k \sum_{i=1}^{l} \bar{s}_{i}\left\|p_{i}-p\right\| .
\end{aligned}
$$

This concludes the proof.

\section{Lemma 3.}

Let $v(p)$ be a function defined on $P, v^{\prime \prime}(p)$ exists and satisfies the condition of Theorem 3. For any $p \in P$ and every convex combination $\sum_{i=1}^{l} \varphi_{i} p_{i}=p$, $\begin{aligned}\left(p_{i} \in P, \varphi_{i}>0, i=1\right. & \left., \ldots, l, \sum_{i=1}^{l} \varphi_{i}=1\right), \\ & v(p)-\sum_{i=1}^{l} \varphi_{i} v\left(p_{i}\right) \geq \frac{\eta}{2 k} \sum_{i=1}^{l} \varphi_{i}\left\|p-p_{i}\right\|^{2} .\end{aligned}$ 
Proof.

For the purpose of this proof only, for any $p=\left(p^{1}, \ldots, p^{k}\right) \in P$ we denote by $\hat{p}$ the vector $\left(p^{1}, \ldots, p^{k-1}\right) \in E^{k-1}$. It is easily verified that if $p$ and $q$ are in $P$, then $\|\hat{p}-\hat{q}\|^{2} \geq \frac{1}{k}\|p-q\|^{2}$.

For any $p_{i}, i=1, \ldots, l$ let $u_{i}=\left(\hat{p}-\hat{p}_{i}\right) /\left\|\hat{p}-\hat{p}_{i}\right\|$. (Since $\varphi_{i}>0, \hat{p}-\hat{p}_{i} \neq 0$.) By Taylor's theorem we have:

$$
v\left(p_{i}\right)=v(p)+\left(\hat{p}_{i}-\hat{p}\right) v^{\prime}(p)+\frac{1}{2}\left(\hat{p}_{i}-\hat{p}\right) v^{\prime \prime}\left(\xi_{i}\right)\left(\widetilde{\hat{p}_{i}-\hat{p}}\right)
$$

where $\xi_{i}=p_{i}+\alpha_{i}\left(p-p_{i}\right)$ for some $\alpha_{i} ; 0 \leq \alpha_{i} \leq 1$. Hence:

$$
v\left(p_{i}\right)=v(p)+\left(\hat{p}_{i}-\hat{p}\right) v^{\prime}(p)+\frac{1}{2}\left\|\hat{p}-\hat{p}_{i}\right\|^{2} u_{i} v^{\prime \prime}\left(\xi_{i}\right) \tilde{u}_{i} .
$$

Since $\left\|u_{i}\right\|=1$ we get:

$$
v\left(p_{i}\right) \leq v(p)+\left(\hat{p}_{i}-\hat{p}\right) v^{\prime}(p)-\frac{\eta}{2 k}\left\|p-p_{i}\right\|^{2} .
$$

Now (12) is obtained by multiplying the last equation by $\varphi_{i}$ and summing on $i$, (recalling that $\Sigma_{1}^{l} \varphi_{i}=1$ and $\Sigma_{1}^{l} \varphi_{i}\left(\hat{p}_{i}-\hat{p}\right)=0$ ).

Proof of Theorem 3.

Since it is well known that $\sum_{r=1}^{n} \frac{1}{r}=0(\log n)$, the theorem will follow from the inequality:

$$
v_{n}(p) \leq v(p)+\frac{k_{n}}{n}, \quad p \in P \text { for } n=1,2, \ldots
$$

where $k_{n}=\sum_{r=1}^{n-1} \frac{\alpha}{r}, n=2,3, \ldots k_{1}$ and $\alpha$ being constants.

We prove (13) by induction on $n$ : Choose $k_{1}$ large enough to make (13) true for $n=1$. Let $\alpha=\operatorname{Max}\left(k_{1}, \frac{k c^{2}}{2 \eta}\right)$ where $c$ is the constant determined by Lemma 2 . By (1) and Lemma 2, we have:

$$
v_{n+1}(p) \leq \frac{1}{n+1} \max _{\sigma^{*} \in \Sigma^{*}}\left\{u(p)+c \sum_{i=1}^{l} \bar{s}_{i}\left\|p-p_{i}\right\|+n \sum_{i=1}^{l} \bar{s}_{i} v_{n}\left(p_{i}\right)\right\} .
$$

Assuming now that (13) is true for $n$ and using the fact that $u(p) \leq v(p)$ we obtain:

$$
\begin{aligned}
v_{n+1}(p) & \leq \frac{1}{n+1} \max _{\sigma^{*} \in \Sigma^{*}}\left\{v(p)+c \sum_{i=1}^{l} \bar{s}_{i}\left\|p-p_{i}\right\|+n \sum_{i=1}^{l} \bar{s}_{i}\left(v\left(p_{i}\right)+\frac{k_{n}}{n}\right)\right\} \\
& =\frac{1}{n+1} \max _{\sigma^{*} \in \Sigma^{*}}\left\{(n+1) v(p)+k_{n}+c \sum_{i=1}^{l} \bar{s}_{i}\left\|p-p_{i}\right\|-n\left(v(p)-\sum_{i=1}^{l} \bar{s}_{i} v\left(p_{i}\right)\right)\right\} .
\end{aligned}
$$

and by Lemma 3 :

$$
v_{n+1}(p) \leq v(p)+\frac{k_{n}}{n+1}+\frac{1}{n+1} \max _{\sigma^{*} \in \Sigma^{*}}\left\{c \sum_{i=1}^{l} \bar{s}_{i}\left\|p-p_{i}\right\|-n \frac{\eta}{2 k} \sum_{i=1}^{l} \bar{s}_{i}\left\|p-p_{i}\right\|^{2}\right\} .
$$


Let $x=\sum_{i=1}^{l} \bar{s}_{i}\left\|p=p_{i}\right\|$, then, by (14), we find directly:

$$
\begin{aligned}
v_{n+1}(p) & \leq v(p)+\frac{k_{n}}{n+1}+\frac{1}{n+1} \max _{x}\left(c x-n \frac{\eta}{2 k} x^{2}\right) \\
& =v(p)+\frac{k_{n}}{n+1}+\frac{1}{n+1} \cdot \frac{k c^{2}}{2 \eta} \cdot \frac{1}{n} \\
& =v(p)+\frac{1}{n+1}\left(k_{n}+\frac{\alpha}{n}\right)=v(p)+\frac{k_{n+1}}{n+1}
\end{aligned}
$$

This completes the proof of (13) and hence the proof of Theorem 3.

5. Games with $\delta_{n}(p)=0^{*}\left(\frac{\log n}{n}\right)$

As an example of a game satisfying the condition of Theorem 3 we consider the following game (which was discussed by AUMANN and MASCHLER): $\sum_{p^{\prime}}^{p} A^{2}$ where $A^{1}=\frac{L}{R}\left(\begin{array}{ll}1 & 0 \\ 0 & 0\end{array}\right)$ and $A^{2}=\begin{aligned} & L \\ & R\end{aligned}\left(\begin{array}{ll}0 & 0 \\ 0 & 1\end{array}\right)$. The game $\Delta(p)$ is $\left(\begin{array}{ll}p & 0 \\ 0 & p^{\prime}\end{array}\right)$ and therefore: $u(p)=p p^{\prime} ; 0 \leq p \leq 1$. Since this is already a concave function we have by Theorem 1: $v(p)=u^{*}(p)=u(p)=p p^{\prime} ; 0 \leq p \leq 1$. For $k=2$, as $v(p)$ is written as a function of a variable $p$, the condition of Theorem 3 is simply $v^{\prime \prime}(p) \leq-\eta<0$. This condition is clearly satisfied in our example since $v^{\prime \prime}(p)=-2$, and hence we have by Theorem 3:

where

$$
v_{n}(p)=p p^{\prime}+\delta_{n}(p) \text { for } 0 \leq p \leq 1, \quad n=1,2, \ldots,
$$

$$
\delta_{n}(p)=0\left(\frac{\log n}{n}\right)
$$
We shall now show that $\delta_{n}(p)$ is in fact of the order of $\left(\frac{\log n}{n}\right)$ and not smaller
than that.

\section{Theorem 4.}

In the above example $\delta_{n}(p)=0^{*}\left(\frac{\log n}{n}\right)$.

To prove the theorem it is sufficient to prove the following proposition:

\section{Proposition.}

In the above example

$$
v_{n}(p) \geq p p^{\prime}+\frac{p p^{\prime}}{n}\left(B_{n}-A_{n}\right), \text { for } 0 \leq p \leq 1, \quad n=1,2, \ldots,
$$


where

$$
\left\{\begin{array}{l}
A_{1}=B_{1}=0 \\
A_{n}=\frac{1}{8} \sum_{k=1}^{n-1} \frac{1}{k^{2}} \\
B_{n}=\frac{1}{4} \sum_{k=1}^{n-1}\left(\frac{1}{k}-\frac{1}{4 k^{2}} \sum_{r=1}^{k-1} \frac{1}{r}\right) ; n=2,3, \ldots
\end{array}\right.
$$

Since $\sum_{k=1}^{n-1} \frac{1}{k^{2}}$ and $\sum_{k=1}^{n-1} \frac{1}{k^{2}} \sum_{r=1}^{k-1} \frac{1}{r}$ are bounded and it is also well known that $\sum_{k=1}^{n-1} \frac{1}{k}=0^{*}(\log n)$, it follows that $B_{n}=0^{*}(\log n)$ and therefore $\frac{p p^{\prime}}{n}\left(B_{n}-A_{n}\right)=$ $0^{*}\left(\frac{\log n}{n}\right)$ which proves the theorem since we have also $\delta_{n}(p)=0\left(\frac{\log n}{n}\right)$.

Proof of the proposition.

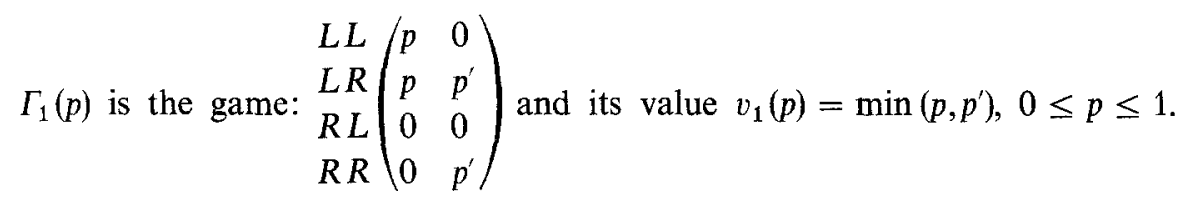

So for $n=1, v_{1}(p) \geq p p^{\prime}$ in accordance to (15). We proceed by induction on $n$. Assume (15) is true for $n$. Since for $p p^{\prime}=0(15)$ is trivially true for $n+1$ we assume $p \neq 1$ and $p \neq 0$, then by Lemma 1 (using the same notations as in Section 3)

$$
v_{n+1}(p)=\frac{1}{n+1} \max _{\substack{0 \leq s \leq 1 \\ 0 \leq t \leq 1}}\left\{\min \left(p s, p^{\prime} t^{\prime}\right)+n\left(\bar{s} v_{n}\left(p_{L}\right)+\bar{s}^{\prime} v_{n}\left(p_{R}\right)\right)\right\}
$$

Restricting the maximization domain by the additional condition $p s=p^{\prime} t^{\prime}$ we get by (5):

and so by (17):

$$
\bar{s}=p^{\prime}, \quad \bar{s}^{\prime}=p, \quad p_{L}=\frac{p s}{p^{\prime}} \quad \text { and } \quad p_{R}=s^{\prime},
$$

$$
v_{n+1}(p) \geq \frac{1}{n+1} \max _{\substack{0 \leq s \leq 1 \\ p s / p^{\prime} \leq 1}}\left\{p s+n\left(p^{\prime} v_{n}\left(\frac{p s}{p^{\prime}}\right)+p v_{n}\left(s^{\prime}\right)\right)\right\}
$$

Using the induction hypothesis we obtain:

$$
\begin{aligned}
& v_{n+1}(p) \geq \frac{1}{n+1} \max _{\substack{0 \leq s \leq 1 \\
p s / p^{\prime} \leq 1}}\{p s+n p^{\prime}\left[\frac{p s}{p^{\prime}}\left(1-\frac{p s}{p^{\prime}}\right)\left(1+\frac{B_{n}}{n}\right)-\frac{A_{n}}{n}\right] \\
&\left.+n p\left[s(1-s)\left(1+\frac{B_{n}}{n}\right)-\frac{A_{n}}{n}\right]\right\} .
\end{aligned}
$$


By simplifying the expression:

where:

$$
v_{n+1}(p) \geq \frac{1}{n+1}\left(\max _{\substack{0 \leq s \leq 1 \\ p s / p^{\prime} \leq 1}} \varphi(s)-A_{n}\right)
$$

$$
\varphi(s)=p\left(1+2\left(n+B_{n}\right)\right) s-\frac{p}{p^{\prime}}\left(n+B_{n}\right) s^{2} .
$$

The parabola $\varphi(s)$ has a maximum at $s_{0}=p^{\prime}\left(1+\frac{1}{2\left(n+B_{n}\right)}\right)$ and:

$$
\varphi\left(s_{0}\right)=p p^{\prime}\left((n+1)+B_{n}+\frac{1}{4\left(n+B_{n}\right)}\right) .
$$

We shall show now that:

$$
B_{n+1} \leq B_{n}+\frac{1}{4\left(n+B_{n}\right)}
$$

In fact since $0 \leq \frac{B_{n}}{n}<1$ we have

$$
\begin{aligned}
\frac{1}{n+B_{n}} & \geq \frac{1}{n}\left(1-\frac{B_{n}}{n}\right)=\frac{1}{n}\left(1-\frac{1}{4 n} \sum_{k=1}^{n-1}\left(\frac{1}{k}-\frac{1}{4 k^{2}} \sum_{r=1}^{k-1} \frac{1}{r}\right)\right) \\
& \geq \frac{1}{n}\left(1-\frac{1}{4 n} \sum_{k=1}^{n-1} \frac{1}{k}\right)=4\left(B_{n+1}-B_{n}\right)
\end{aligned}
$$

which proves (21), (20) and (21) imply

$$
\varphi\left(s_{0}\right) \geq p p^{\prime}\left(n+1+B_{n+1}\right) .
$$

Consider now the two cases:

\section{Case a.}

$s_{0} \leq 1$ and $\frac{p s_{0}}{p^{\prime}} \leq 1$ which means that $s_{0}$ is in the domain of the maximization in (18) and therefore by (18) and (22):

$$
\begin{aligned}
v_{n+1}(p) & \geq \frac{1}{n+1}\left(\varphi\left(s_{0}\right)-A_{n}\right) \geq \frac{p p^{\prime}}{n+1}\left(n+1+B_{n+1}-A_{n}\right) \\
& \geq p p^{\prime}+\frac{p p^{\prime}}{n+1}\left(B_{n+1}-A_{n+1}\right) .
\end{aligned}
$$

This completes the induction step for this case.

Case b.

$$
s_{0}>1 \text { or } \frac{p s_{0}}{p^{\prime}}>1 \text { (i.e. } s_{0} \text { is out of the domain of the maximization in (18)). }
$$


We claim that in this case:

$$
p p^{\prime}<\frac{1}{2\left(n+B_{n}\right)}
$$

In fact:

$$
\begin{gathered}
s_{0}>1 \Rightarrow p^{\prime}\left(1+\frac{1}{2\left(n+B_{n}\right)}\right)>1 \Rightarrow p<\frac{1}{1+2\left(n+B_{n}\right)} \Rightarrow p p^{\prime}<\frac{1}{2\left(n+B_{n}\right)} \\
\frac{p s_{0}}{p^{\prime}}>1 \Rightarrow p\left(1+\frac{1}{2\left(n+B_{n}\right)}\right)>1 \Rightarrow p^{\prime}<\frac{1}{1+2\left(n+B_{n}\right)} \Rightarrow p p^{\prime}<\frac{1}{2\left(\mathrm{n}+B_{n}\right)} .
\end{gathered}
$$

We notice that $\varphi\left(p^{\prime}\right)=p p^{\prime}\left(n+1+B_{n}\right)$. Applying (23) we obtain:

$$
\begin{aligned}
\varphi\left(p^{\prime}\right) & =p p^{\prime}\left(n+1+B_{n}+\frac{1}{4\left(n+B_{n}\right)}\right)-\frac{p p^{\prime}}{4\left(n+B_{n}\right)} \\
& =\varphi\left(s_{0}\right)-\frac{p p^{\prime}}{4\left(n+B_{n}\right)} \geq \varphi\left(s_{0}\right)-\frac{1}{8\left(n+B_{n}\right)^{2}} \geq \varphi\left(s_{0}\right)-\frac{1}{8 n^{2}} .
\end{aligned}
$$

Inserting this in (18) gives:

$$
\begin{aligned}
v_{n+1}(p) & \geq \frac{1}{n+1}\left(\varphi\left(p^{\prime}\right)-A_{n}\right) \geq \frac{1}{n+1}\left(\varphi\left(s_{0}\right)-\left(A_{n}+\frac{1}{8 n^{2}}\right)\right) \\
& =\frac{1}{n+1}\left(\varphi\left(s_{0}\right)-A_{n+1}\right) \geq p p^{\prime}+\frac{p p^{\prime}}{n+1}\left(B_{n+1}-A_{n+1}\right) .
\end{aligned}
$$

This completes the proof of the proposition and hence also the proof of Theorem 4.

Note that the proof of the proposition suggests a constructive way to calculate a strategy for player $I$ in $\Gamma_{n}(p)$ that guarantees him an error term of the highest order of magnitude possible. (Namely, calculate $s_{0}$, consider the two cases etc.) We may call such a strategy "essentially optimal".

\section{Games with $\delta_{n}=0^{*}(1 / n)$}

In this section we introduce a family of games for which $\delta_{n}=0^{*}(1 / n)$. For such games the sum of the total payoff to player I beyond $v(p)$, in all the stages of the game, is bounded.

\section{Theorem 5.}

Let $\sum_{p^{k}}^{p^{1}-A_{A^{k}}^{1}} A^{1}$ be a repeated game with incomplete information in which $\frac{v(p)-u(p)}{\sum_{v=1}^{k}\left(p^{v}\left(1-p^{v}\right)\right)^{3 / 2}} \geq \varepsilon>0$ for every $p$ in $P$ which is not an extreme point, then: 


$$
v_{n}(p) \leq v(p)+\frac{\alpha \sum_{\nu=1}^{k} \sqrt{p^{v}\left(1-p^{v}\right)}}{n} ; \quad p \in P,
$$

for $n=1,2, \ldots$ where $\alpha$ is a constant.

The theorem follows easily from the following two lemmas:

\section{Lemma 4.}

For any $p \in P$ which is not an extreme point of $P$ and for any $\sigma^{*}=\left(\sigma^{1}, \ldots, \sigma^{k}\right) \in \Sigma^{*}$ we have

where

$$
\underset{1 \leq j \leq m}{\operatorname{Min}} \sum_{v=1}^{k} p^{v} \sigma^{v} A^{v} \mathrm{e}_{j} \leq u(p)+a \sum_{v=1}^{k} p^{v}\left(1-p^{v}\right) \sum_{i=1}^{l}\left|s_{i}^{v}-s_{i}^{v *}\right|
$$

$$
s_{i}^{v *}=\sum_{\substack{\rho=1 \\ \rho \neq \nu}}^{k} \frac{p^{\rho}}{1-p^{v}} s_{i}^{\rho} ; \quad v=1, \ldots, k ; \quad i=1, \ldots, l
$$

and $a=\max \left\{\left|a_{i j}^{v}\right| \mid 1 \leq i \leq l, 1 \leq j \leq m, 1 \leq v \leq k\right\}\left(s_{i}^{v *}\right.$ is well defined since $p$ is not an extreme point of $P$ ).

\section{Proof.}

By (11) in the proof of Lemma 2:

$$
\begin{aligned}
\min _{1 \leq j \leq m} \sum_{\nu=1}^{k} p^{v} \sigma^{v} A^{v} \mathrm{e}_{j} & \leq u(p)+a \sum_{\nu=1}^{k} p^{v} \sum_{i=1}^{l}\left|s_{i}^{v}-\bar{s}_{i}\right| \\
& =u(p)+a \sum_{\nu=1}^{k} p^{v} \sum_{i=1}^{l}\left|s_{i}^{v}-\sum_{\rho=1}^{k} p^{\rho} s_{i}^{\rho}\right| \\
& =u(p)+a \sum_{\nu=1}^{k} p^{\nu} \sum_{i=1}^{l}\left|\left(1-p^{\nu}\right) s_{i}^{\nu}-\sum_{\substack{\rho=1 \\
\rho \neq v}}^{k} p^{\rho} s_{i}^{\rho}\right| \\
& =u(p)+a \sum_{\nu=1}^{k} p^{\nu}\left(1-p^{\nu}\right) \sum_{i=1}^{l}\left|s_{i}^{\nu}-s_{i}^{\nu *}\right| .
\end{aligned}
$$

\section{Lemma 5.}

For any $p \in P$ and $\sigma^{*}=\left(\sigma^{1}, \ldots, \sigma^{k}\right) \in \Sigma^{*}$ the following inequality holds:

$$
\sum_{i=1}^{l} \bar{s}_{i} \sqrt{p_{i}^{v}\left(1-p_{i}^{\nu}\right)} \leq \sqrt{p^{v}\left(1-p^{v}\right)}\left(1-\frac{1}{8} \sum_{i=1}^{l}\left|s_{i}^{v}-s_{i}^{v *}\right|^{2}\right)
$$

for $v=1, \ldots, k$, where $\bar{s}_{i}$ and $p_{i}$ are defined by (2) and $s_{i}^{v *}$ is defined by (25).

\section{Proof.}

If $p$ is an extreme point of $P$, inequality (26) is clearly true; if this is not the case, we recall our notation that all summations over $i$ are only over those $i$ 's for which $\bar{s}_{i} \neq 0$, so that by (2) we obtain: 


$$
\begin{aligned}
\sum_{i=1}^{l} \bar{s}_{i} \sqrt{p_{i}^{v}\left(1-p_{i}^{v}\right)} & =\sum_{i=1}^{l} \bar{s}_{i} \sqrt{\frac{p^{v} s_{i}^{v}}{\bar{s}_{i}}\left(1-\frac{p^{v} s_{i}^{v}}{\bar{s}_{i}}\right)}=\sum_{i=1}^{l} \sqrt{p^{v} s_{i}^{v}\left(\bar{s}_{i}-p^{v} s_{i}^{v}\right)} \\
& =\sum_{i=1}^{l} \sqrt{p^{v} s_{i}^{v}\left(\sum_{\rho=1}^{k} p^{\rho} s_{i}^{\rho}-p^{v} s_{i}^{v}\right)}=\sum_{i=1}^{l} \sqrt{p^{v} s_{i}^{v} \sum_{\rho \neq v} p^{\rho} s_{i}^{\rho}} \\
& =\sum_{i=1}^{l} \sqrt{p^{v} s_{i}^{v}\left(1-p^{v}\right) \sum_{\rho \neq \nu} \frac{p^{\rho}}{1-p^{v}} s_{i}^{\rho}}=\sqrt{p^{v}\left(1-p^{v}\right)} \sum_{i=1}^{l} \sqrt{s_{i}^{v} s_{i}^{v *}} \\
& =\sqrt{p^{v}\left(1-p^{v}\right)} \sum_{i=1}^{l}\left(\frac{s_{i}^{v}+s_{i}^{v *}}{2}-\frac{\left(\sqrt{s_{i}^{v}}-\sqrt{\left.s_{i}^{v *}\right)^{2}}\right.}{2}\right)
\end{aligned}
$$

Now $\left|\sqrt{s_{i}^{v}}-\sqrt{s_{i}^{v *}}\right| \geq \frac{\left|s_{i}^{v}-s_{i}^{v *}\right|}{2}$. This is trivial if $s_{i}^{v}=s_{i}^{v *}=0$ otherwise:

$$
\left|\sqrt{s_{i}^{v}}-\sqrt{s_{i}^{v *}}\right|=\frac{\left|s_{i}^{v}-s_{i}^{v *}\right|}{\sqrt{s_{i}^{v}}+\sqrt{s_{i}^{v *}}} \geq \frac{\left|s_{i}^{v}-s_{i}^{\nu *}\right|}{2} .
$$

Hence noticing that $\sum_{i=1}^{l} s_{i}^{v}=\sum_{i=1}^{l} s_{i}^{v *}=1$ we obtain finally:

$$
\sum_{i=1}^{l} \bar{s}_{i} \sqrt{p_{i}^{v}\left(1-p_{i}^{v}\right)} \leq \sqrt{p^{v}\left(1-p^{v}\right)}\left(1-\frac{1}{8} \sum_{i=1}^{l}\left|s_{i}^{v}-s_{i}^{\nu *}\right|^{2}\right) \text {. }
$$

\section{Proof of Theorem 5.}

If $p$ is an extreme point of $P$ then, and only then, $\sum_{i=1}^{l} p^{v}\left(1-p^{v}\right)=0$ and (24) is trivially true for any $\alpha$. Assume from now on that $\sum_{\nu=1}^{k} p^{v}\left(1-p^{v}\right) \neq 0$. Choose $\alpha>0$ such that (24) holds for $n=1$, and such that $\alpha \geq \frac{2 c^{2}}{\varepsilon}$ where $c$ is the constant determined by Lemma 2. For such an $\alpha$ we now prove (24) by induction on $n$. For $n=1$ it is true by definition of $\alpha$. Assume it is true for $n$ and let us prove it for $n+1$. By Lemma 1 und Lemma 4 we have:

$$
v_{n+1}(p) \leq \frac{1}{n+1} \max _{\sigma^{*} \in \Sigma^{*}}\left\{u(p)+a \sum_{\nu=1}^{k} p^{\nu}\left(1-p^{v}\right) \sum_{i=1}^{l}\left|s_{i}^{v}-s_{i}^{v *}\right|+n \sum_{i=1}^{l} \bar{s}_{i} v_{n}\left(p_{i}\right)\right\} \text {. }
$$

Applying the induction hypothesis we have:

$$
\begin{aligned}
v_{n+1}(p) \leq \frac{1}{n+1} \max _{\sigma^{*} \in \Sigma^{*}}\{u(p) & +a \sum_{v=1}^{k} p^{v}\left(1-p^{v}\right) \sum_{i=1}^{l}\left|s_{i}^{v}-s_{i}^{\nu *}\right|+n v(p) \\
& \left.+\alpha \sum_{i=1}^{l} \bar{s}_{i} \sum_{v=1}^{k} \sqrt{p_{i}^{v}\left(1-p_{i}^{v}\right)}\right\}
\end{aligned}
$$


Let $x^{\nu}=\max _{1 \leq i \leq l}\left|s_{i}^{\nu}-s_{i}^{\nu *}\right|$ then by Lemma 5 :

$$
\begin{aligned}
\sum_{i=1}^{l} \bar{s}_{i} \sqrt{p_{i}^{v}\left(1-p_{i}^{v}\right)} & \leq \sqrt{p^{v}\left(1-p^{v}\right)}\left(1-\frac{1}{8} \sum_{i=1}^{l}\left|s_{i}^{v}-s_{i}^{v *}\right|^{2}\right) \\
& \leq \sqrt{p^{v}\left(1-p^{v}\right)}\left(1-\frac{1}{8}\left(x^{v}\right)^{2}\right)
\end{aligned}
$$

on the other hand $a \sum_{i=1}^{l}\left|s_{i}^{\nu}-s_{i}^{\nu *}\right| \leq a k x^{\nu}=c x^{\nu}$. So by (27):

$$
\begin{aligned}
v_{n+1}(p) \leq v(p) & +\frac{1}{n+1} \max _{\sigma^{*} \in \Sigma^{*}}\left\{u(p)-v(p)+c \sum_{\nu=1}^{k} p^{v}\left(1-p^{v}\right) x^{\nu}\right. \\
& \left.+\alpha \sum_{\nu=1}^{k} \sqrt{p^{v}\left(1-p^{v}\right)}\left(1-\frac{1}{8}\left(x^{\nu}\right)^{2}\right)\right\}
\end{aligned}
$$

which is

$$
\begin{aligned}
v_{n+1}(p) \leq v(p)+\frac{\alpha \sum_{\nu=1}^{k} \sqrt{p^{v}\left(1-p^{v}\right)}}{n+1}+\{u(p) & -v(p)+\max _{\sigma^{*} \in \Sigma^{*}} \sum_{v=1}^{k}\left(c p^{v}\left(1-p^{\nu}\right) x^{v}\right. \\
& \left.\left.-\frac{\alpha}{8} \sqrt{p^{\nu}\left(1-p^{v}\right)}\left(x^{\nu}\right)^{2}\right)\right\} .
\end{aligned}
$$

Now

$$
\begin{gathered}
\max _{\sigma^{*} \in \Sigma^{*}} \sum_{v=1}^{k}\left(c p^{v}\left(1-p^{v}\right) x^{v}-\frac{\alpha}{8} \sqrt{p^{v}\left(1-p^{v}\right)}\left(x^{v}\right)^{2}\right) \\
\leq \sum_{v=1}^{k} \max _{x^{v}}\left(c p^{v}\left(1-p^{v}\right) x^{v}-\frac{\alpha}{8} \sqrt{p^{v}\left(1-p^{v}\right)}\left(x^{v}\right)^{2}\right) \\
=\sum_{v=1}^{k} \frac{2 c^{2}}{\alpha}\left(p^{v}\left(1-p^{v}\right)\right)^{3 / 2} .
\end{gathered}
$$

So by definition of $\alpha$ and by $\frac{v(p)-u(p)}{\sum_{v=1}^{k}\left(p^{v}\left(1-p^{v}\right)\right)^{3 / 2}} \geq \varepsilon$ we have

$$
\begin{aligned}
u(p)-v(p) & +\max _{\sigma^{*} \in \Sigma^{*}} \sum_{\nu=1}^{k}\left(c p^{v}\left(1-p^{v}\right) x^{v}-\frac{\alpha}{8} \sqrt{p^{v}\left(1-p^{v}\right)}\left(x^{\nu}\right)^{2}\right) \\
& \leq \frac{2 c^{2}}{\alpha} \sum_{\nu=1}^{k}\left(p^{v}\left(1-p^{v}\right)\right)^{3 / 2}-(v(p)-u(p)) \\
& \leq \varepsilon \sum_{\nu=1}^{k}\left(p^{v}\left(1-p^{v}\right)\right)^{3 / 2}-(v(p)-u(p)) \leq 0 .
\end{aligned}
$$
Hence by $(28)$ we have finally $v_{n+1}(p) \leq v(p)+\frac{\alpha \sum_{v=1}^{k} \sqrt{p^{v}\left(1-p^{v}\right)}}{n+1}$ which com-
letes the proof of the theorem. 


\section{Example 1.}

Consider the game:

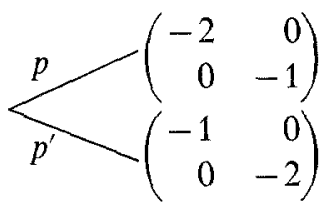

$\Delta(p)$ is the game $\left(\begin{array}{cc}-(1+p) & 0 \\ 0 & -\left(1+p^{\prime}\right)\end{array}\right)$ and its value is $u(p)=-\frac{2}{3}-\frac{p p^{\prime}}{3}$. Hence by Theorem 1: $v(p)=u^{*}(p)=-\frac{2}{3}$ for $0 \leq p \leq 1$. Now for this special case as $k=2$ we have:

$$
\frac{u(p)-u(p)}{\sum_{v=1}^{k}\left(p^{v}\left(1-p^{v}\right)\right)^{3 / 2}}=\frac{v(p)-u(p)}{2\left(p p^{\prime}\right)^{3 / 2}}=\frac{1}{6\left(p p^{\prime}\right)^{1 / 2}} \geq \frac{1}{3}
$$

Hence, by Theorem $5, v_{n}(p) \leq-\frac{2}{3}+\frac{\alpha \sqrt{p p^{\prime}}}{n}$ for $0 \leq p \leq 1$ and $n=1,2, \ldots$.

Proposition.

$$
\begin{gathered}
v_{n}(p) \geq-\frac{2}{3}+\frac{f(p)}{n} \text { for } 0 \leq p \leq 1 \text { and } n=1,2, \ldots \text { where } \\
f(p)= \begin{cases}\frac{p}{3} & 0 \leq p<\frac{1}{2} \\
\frac{p^{\prime}}{3} & \frac{1}{2} \leq p \leq 1 .\end{cases}
\end{gathered}
$$

First it is easily verified that $v_{1}(p)=-\frac{2}{3}+f(p)$. Assume that the inequality is true for $n$ and let $\left(\left(s, s^{\prime}\right) ;\left(t, t^{\prime}\right)\right)$ be an optimal strategy for player $\mathrm{I}$ in $\Gamma_{1}(p)$. Using this strategy in the first stage of $\Gamma_{n+1}(p)$ player I guarantees (by the recursion formula (1)): $\frac{1}{n+1}\left\{v_{1}(p)+n\left(\bar{s} v_{n}\left(p_{L}\right)+\bar{s}^{\prime} v_{n}\left(p_{R}\right)\right)\right\}$ which is by the induction hypothesis at least:

$$
\frac{1}{n+1}\left(v_{1}(p)-n \cdot \frac{2}{3}\right)=-\frac{2}{3}+\frac{f(p)}{n+1} .
$$

We conclude that in the game under consideration $\delta_{n}=0 *\left(\frac{1}{n}\right)$.

\section{Example 2.}

Consider the game:

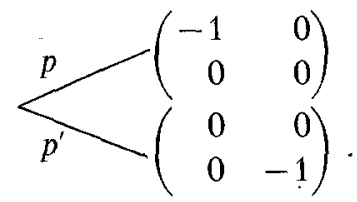

In this case $\Delta(p)$ is the game $\left(\begin{array}{rr}-p & 0 \\ 0 & -p^{\prime}\end{array}\right)$ and its value is $u(p)=-p p^{\prime}$. Hence, 
by Theorem 1: $v(p)=u^{*}(p)=0$ for $0 \leq p \leq 1 ; \frac{v(p)-u(p)}{2\left(p p^{\prime}\right)^{3 / 2}}=\frac{1}{2 \sqrt{p p^{\prime}}} \geq \frac{1}{4}$ and again, by Theorem 5 , we deduce $v_{n}(p)=v(p)+\delta_{n}(p)$, where $\delta_{n}(p) \leq \frac{\alpha \sqrt{p p^{\prime}}}{n}$. In this case $\alpha=0$ (and therefore $\delta_{n}(p)=0$ for $0 \leq p \leq 1, n=1,2, \ldots$ ). This easily follows from the fact that $v_{1}(p)=0$.

\section{Discussion}

The main result of this paper is given by Theorem 2 which proves the existence of games with error term of the order of $1 / \sqrt{n}$, the highest order possible according to MerTens and ZAMir and AUMANN and MASCHLER.

We denote by $p_{i}(i=1, \ldots, n)$ the conditional probability assigned by player II, at the end of stage $i$, to the event "chance has chosen $A^{1}$ ". It was shown both by MERTENS and ZAMIR and by AUMANN and MASCHLER that given the strategy of player I, $\left\{p_{i}\right\}$ is a Martingale and $v_{n}(p) \leq v(p)+\frac{c}{n} E\left(\sum_{i=1}^{n}\left|p_{i+1}-p_{i}\right|\right)$, where $c$ is a constant and $E$ denotes the expectation. By this inequality we deduce the following corollary of Theorem 2 :

Corollary.

There exists a constant $c>0$ such that for any $n, n=1,2, \ldots$, and for any $p_{1}$, $0 \leq p_{1} \leq 1$, there exists a Martingale $\left\{p_{i}\right\} ; 0 \leq p_{i} \leq 1 ; i=1, \ldots, n$ such that:

$$
E\left(\sum_{i=1}^{n}\left|p_{i+1}-p_{i}\right|\right) \geq c p_{1}\left(1-p_{1}\right) \sqrt{n} .
$$

This is an interesting result in itself, since it is well known that for any bounded Martingale $\left\{p_{i}\right\} ; E\left(\sum_{i=1}^{n}\left|p_{i+1}-p_{i}\right|\right)=0(\sqrt{n})$.

A second important result in the present paper is the inductive construction of "essentially optimal" strategies for player $\mathrm{I}$ in $\Gamma_{n}(p)$ suggested in the proofs of Theorems 2 and 4 . Though by some simple arguments it is possible to find the error terms of some games, which do not satisfy the conditions of Theorems 3 and 5, there does not exist a general method of determining the error term of any given game. We conclude this paper by an example of a game whose error term cannot be found by the theorems stated in this paper, and in fact we do not know its error term:

Consider the game

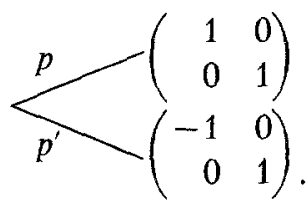


For this game,

$$
u(p)= \begin{cases}0 & 0 \leq p \leq \frac{1}{2} \\ 1-\frac{1}{2 p} & \frac{1}{2} \leq p \leq 1,\end{cases}
$$

and hence by Theorem 1:

$$
v(p)=u^{*}(p)=\frac{p}{2}
$$

We can show that $\delta_{n}(p) \leq k \frac{\sqrt{p p^{\prime}} \sqrt[4]{n}}{n}$, and our conjecture is that $\delta_{n}(p)=0^{*}(1 / n)$.

\section{References}

Aumann, R. J., and M. MaschleR: Game Theoretic Aspects of Gradual Disarmament. Report to the U.S. A.C.D.A. (Arms Control and Disarmament Agency, Washington, D. C.) Final report on Contract ACDA/ST-80, prepared by Mathematica, Princeton N.J. Chapter V, June 1966. Harsanyl, J. C.: Games with Incomplete Information Played by Bayesian Players. Parts I, II, III. Management Science, 14, No. 3, 5, 7, 1967-1968.

MERTENS, J.-F., and S. ZAMIR: The value of two-person zero-sum repeated games with lack of information on both sides. International Journal of Game Theory, 1, No. 1, 1971. 